Preprint of: Müller, AM, Khoo, S. \& Lambert, R. (2013). Review of Physical Activity Prevalence of Asian School-age Children and Adolescents. Asian-Pacific Journal of Public Health, 26 (3), 227-238. doi: 10.1177/1010539513481494. Visit the following link for more information: http://aph.sagepub.com/content/25/3/227.short

\title{
Review of Physical Activity Prevalence of Asian School-Age Children and Adolescents
}

\author{
A Müller (M.A.) ${ }^{1}$, S Khoo (PhD) ${ }^{2}$ and R Lambert (M.A.) $)^{1}$ \\ ${ }^{1}$ College of Physical Education, The University of Suwon, South Korea \\ ${ }^{2}$ Sports Centre, University of Malaya, Kuala Lumpur, Malaysia
}

Email for correspondence:

André Matthias Müller, M.A.

Email: andrematthiasmueller@gmail.com 


\title{
Review of Physical Activity Prevalence of Asian School-Age Children and Adolescents
}

\begin{abstract}
Overweight and obese populations in Asia are high and increasing rapidly. Physical activity prevalence studies have found low physical activity rates among Asian school-age children and adolescents. The purpose of this review is to establish a baseline for physical activity rates in Asian school-age children and adolescents and serve as a platform for additional research. Thirty papers published between 2000 and 2011 on physical activity prevalence of school-age children and adolescents were included in the review. Fourteen studies were conducted in East Asia, 10 in South East Asia, 2 in South Asia and 2 in West Asia. Studies used subjective methods (questionnaires or diaries) and objective methods (accelerometers or heart rate monitors). Although nearly all studies found low physical activity levels, it was not possible to provide an accurate picture due to an absence of standardized, reliable, and economically viable measurement tools.
\end{abstract}

Keywords: adolescents, Asia, children, physical activity prevalence, review, school-age 


\section{Introduction}

According to the World Health Organization in 2015, approximately 1.5 billion adults will be overweight with attendant health risks. ${ }^{1}$ An obesity forecast for the USA conducted by Finkelstein et al. ${ }^{2}$ indicates that obesity will increase between $33 \%$ and $51 \%$ until the year 2030. The population gaining excess weight is not limited to just adults in developed and developing nations, ${ }^{3-5}$ but is also a global trend among children and adolescents of both sexes. $^{6,7}$ Wang and Lobstein ${ }^{8}$ compiled overweight and obesity rates from 1980 to 2005 for school-age children in 25 countries and preschool-age children in 42 countries. They found that the prevalence increased in almost all countries except of school-age children in Russia and to some extent in Poland during the 1990s.

Many countries in the Asia-Pacific region show the highest levels and the fastest increase of obesity in the world. ${ }^{9}$ For example, in Vietnam $9.6 \%$ of the adolescent population is obese and $10.9 \%$ are overweight. ${ }^{10}$ Further, $18.3 \%$ of school-age children and adolescents are overweight or obese in Malaysia, ${ }^{11} 20 \%$ of children are overweight or obese in China, ${ }^{12}$ and $11.7 \%$ of male adolescents and $14.7 \%$ of female adolescents in Saudi Arabia are also overweight or obese. ${ }^{13}$

Studies by Laitinen et al. ${ }^{14}$ and Harper ${ }^{15}$ concluded that early obesity is a precursor for undesirable body mass index (BMI) in adulthood and a catalyst for various medical conditions. Obesity is associated with type 2 diabetes mellitus, hypertension and cardiovascular diseases and metabolic syndrome. ${ }^{16,17}$ Metabolic syndrome is noted in only $4 \%$ of normal weight youngsters, but present in $29 \%$ of overweight or obese youngsters. ${ }^{18}$

Costs for the health system associated with obesity and other related diseases in children and adolescents were estimated by Wang and Dietz ${ }^{19}$ in the USA between 1979 and 1999. The annual hospital costs for obesity and its co-morbidities increased more than threefold resulting in a considerable economic burden. A $1 \%$ reduction of the current adult 
obesity rate per annum in the USA would result in USD 84.9 billion savings in the medical sector. $^{2}$

A physically active lifestyle can significantly decrease body fat and obesity in children and adolescents. ${ }^{20}$ Only $9 \%$ of adolescents engaged in 15 hours of activity per week are overweight or obese as compared to $14 \%$ of adolescents whose activity rate is less than one hour per week. ${ }^{21}$ Children who are active for more than 103 minutes per day are five times less likely to get metabolic syndrome compared to children active less than 43 minutes per day. ${ }^{22}$ Physically active children also enjoy lower blood pressure while bone density is modestly increased with only 10 minutes of moderate to vigorous activity two or three times per week. ${ }^{22}$

Although Asia is the largest and most populous continent in the world, most of the information about physical activity (PA) prevalence in youth comes from western countries. ${ }^{23}$ A review of PA prevalence by Sallis et al. ${ }^{24}$ in 2000 was largely western in scope, with more than $80 \%$ of the articles published in the U.S. A more recent review by Horst et al. ${ }^{25}$ in 2007 also revealed only a few studies from Asia.

The lack of studies focused on PA rates of Asian school-age children and adolescents PA rates and the absence of any assessment of those studies is problematic because data is underutilized and its value unrealized. Therefore this paper attempts to compile a literature review to establish baseline information that can generate additional research initiatives as well as awareness in other sectors leading to implementation of focused PA programmes.

\section{Methods}

Selected studies were retrieved between October and December 2011 using the databases PubMed, MEDLINE, Spolit, and SPORTDiscus, to identify articles containing the following keywords and combinations:

a) Physical Activity OR Physical Exercise OR Exercise OR Sport

b) Children OR Adolescents OR Youth OR School-children OR School-age 
c) Asia OR Afghanistan OR Armenia OR Azerbaijan OR ...

The retrieved studies measured PA in school-age children and adolescents (7-19 years) in Asia published in the English language between the years 2000 and 2011. Google Scholar searches were also used, references from published studies were explored, and experts consulted to identify additional articles. Initially 49 articles were found. After reviewing all full papers 19 articles were excluded, resulting in 30 articles for the review. The 19 articles were excluded because the examined age group differed from the target group in this paper ( 8 articles) or when factors other than PA were measured (11 articles). Figure 1 illustrates the inclusion/exclusion process. Articles were excluded only when there was consensus among all authors.

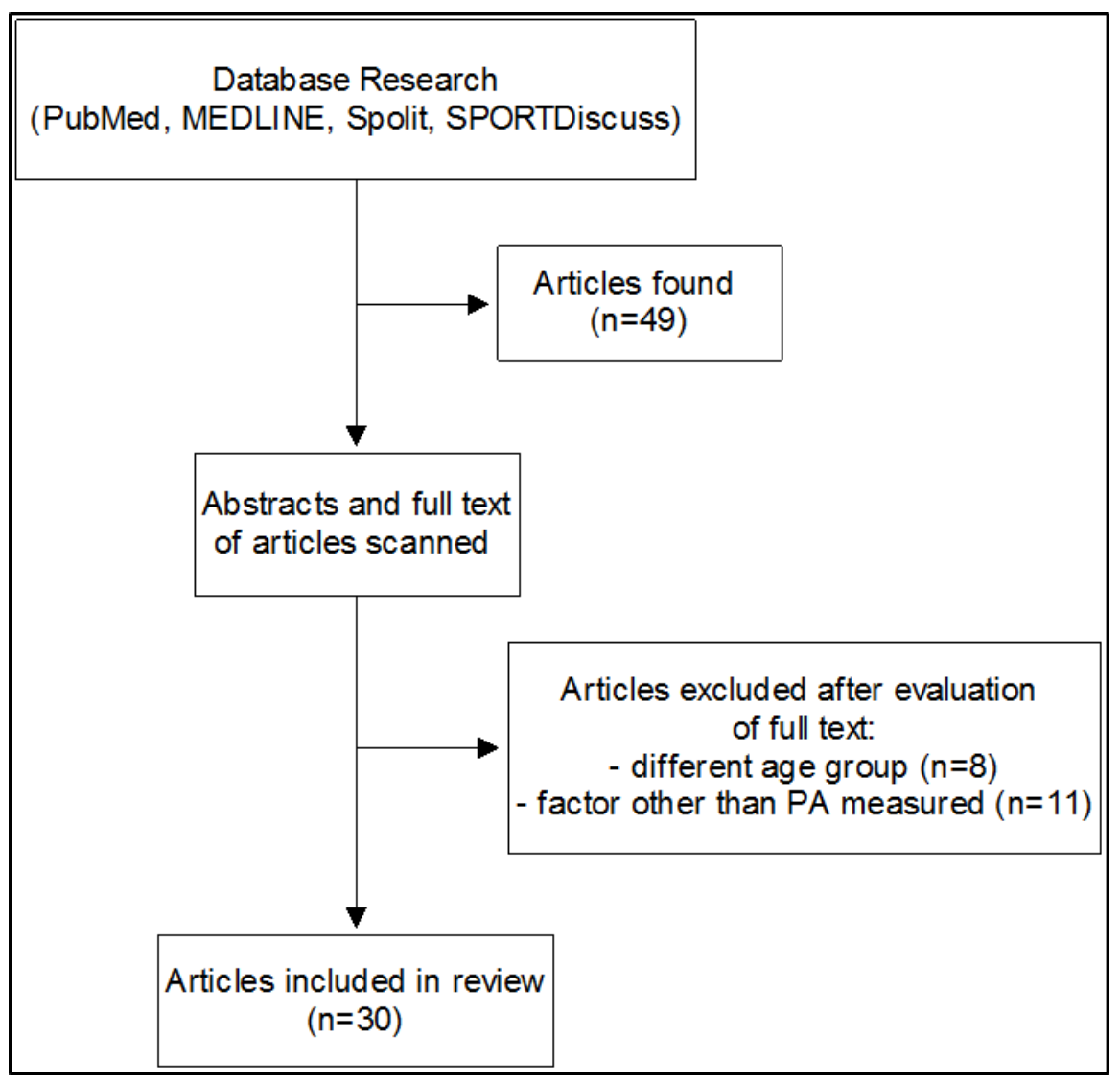

Figure 1. Study inclusion and exclusion process 


\section{Results}

Description of participants

The studies collected data from 38,355 participants between 7 and 19 years old with sample sizes from $30^{26}$ to $6,274^{27}$ About $50.4 \%$ of the participants were female and $49.1 \%$ male. However, 160 participants in the studies by Wang et al. ${ }^{28-30}$ did not indicate their sex. Florentino et al. ${ }^{31}$ and Lachat et al. ${ }^{32}$ did not provide exact figures, but stated there were about equal numbers of male and female participants. Khoo and Al-Shamli ${ }^{33}$ investigated only males, whereas Henry et al. ${ }^{34}$ focused just on females. Of the 30 articles selected, nearly half were conducted in East Asia with 4 studies from China, 4 from Hong Kong, 4 from Taiwan, 1 from South Korea, and 1 study that included Chinese and Korean samples. ${ }^{35}$ South East Asia reflected 3 studies from the Philippines, 4 from Singapore, 2 from Vietnam, and 1 from Malaysia. South Asian studies consisted of 1 from India and 1 from Sri Lanka. Finally, West Asia was represented with 1 study from Israel, 1 from Oman, 1 from Saudi Arabia and 1 from the United Arab Emirates.

\section{Measurement of Physical Activity}

All studies used various objective and subjective measurements. Objective measures of PA included accelerometers or the Double-Labeled Water method (DLW). ${ }^{26}$ Subjective measures used self-report techniques like questionnaires or diaries. Most studies were cross-sectional designs except for the study by Mak and Day ${ }^{36}$ which used a longitudinal design.

As the studies used different international guidelines for PA, five classification levels were created that allowed data to be compared. The levels, ranged from 1-5, are based on recommendations of PA participation for children and adolescents PA. Level 1 indicated the highest recommendation (at least 60min of moderate PA every day) and Level 5 the lowest PA recommendation (at least 30min of moderate PA on at least 3 days per week). Table 1 shows the guidelines for the levels. 
Table 1. PA Guidelines and Levels

\begin{tabular}{ccc}
\hline Reference & Guideline & Level \\
\hline $\begin{array}{c}\text { World Health Organization } \\
(2010)^{37}\end{array}$ & At least 60min moderate PA every day. & 1 \\
$\begin{array}{c}\text { U.S. Department of Health } \\
\text { and Human Service (2005) }\end{array}$ & At least 60min PA on most days in a week. \\
$\begin{array}{c}\text { (4-6d/wk) } \\
\text { Janssen \& LeBlanc }(2010)^{39}\end{array}$ & At least 30min PA every day. \\
$\begin{array}{c}\text { U.S. Department of Health } \\
\text { and Human Service (2000) }\end{array}$ & At least 30min moderate PA on at least 5 \\
days per week. & 3 \\
Strong \& Malina et al. & At least 30min moderate-vigorous PA on at \\
$(2005)^{41}$ & least 3 days per week. \\
\end{tabular}

Data that could not be classified by any of these levels were analyzed by weekly or daily time spent on PA. In studies with multiple PA measuring devices, assessment was based on subjective measures whenever available. Studies that administered self-designed questionnaires or the PAQ-C, ${ }^{41}$ which did not transfer to our classification system, were analyzed separately.

\section{Subjective measures of Physical Activity}

Twenty-six studies used subjective measures whereas four studies included objective and subjective measures. These four studies ${ }^{26,32,42,43}$ are discussed separately. It was only possible to apply the PA guidelines to 15 studies which used subjective measures. These studies are shown in table 2 . 
Table 2. PA Prevalence Based on the Guideline Levels

\begin{tabular}{|c|c|c|c|c|}
\hline Study (country) & $\begin{array}{l}\text { N; Sex; Age } \\
\text { (years) } \pm \text { SD }\end{array}$ & Instrument & Level & Level met $(\%)$ \\
\hline $\begin{array}{l}\text { Chen et al., } 2007 \\
\text { (Taiwan) }^{23}\end{array}$ & $\begin{array}{l}\mathrm{N}=2235 ; \\
\jmath^{\lambda} 1157 / \text { Q } 1078 ; \\
\text { Range: } 12-18\end{array}$ & $\begin{array}{l}\text { Interview questions on } \\
\text { frequency, duration, } \\
\text { type, intensity of PA in } \\
\text { last } 2 \text { weeks. }\end{array}$ & 5 & $28.4 \%$ \\
\hline \multirow{2}{*}{$\begin{array}{l}\text { Chia, } 2008 \\
\text { (Singapore) }^{* 42}\end{array}$} & \multirow{2}{*}{$\begin{array}{l}\mathrm{N}=280 ; \\
\hat{N} 140 / 9139 ; \\
\text { Gp 1: } 10.6 \pm 0.3 \\
\text { Gp 2: } 14.8 \pm 0.6\end{array}$} & \multirow{2}{*}{$\begin{array}{l}\text { The self report PA } \\
\text { Questionnaire (Schmidt } \\
\text { et al., 1998). }\end{array}$} & 2 & $9 \%$ \\
\hline & & & 5 & $30 \%$ \\
\hline $\begin{array}{l}\text { Cho, } 2004 \\
\text { (South Korea) }^{44}\end{array}$ & $\begin{array}{l}\text { Gp 2: } 14.8 \pm 0.6 \\
\mathrm{~N}=1097 ; \\
\precsim 342 / q 755 ; \\
\text { Range: } 13-19\end{array}$ & $\begin{array}{l}\text { PA levels (intensity, } \\
\text { duration, frequency } \\
\text { during leisure time in } \\
\text { last } 4 \text { weeks) }\end{array}$ & 5 & $19.6 \%$ \\
\hline $\begin{array}{l}\text { Chou \& Pei, } \\
2010 \text { (Taiwan) }^{45}\end{array}$ & $\begin{array}{l}\mathrm{N}=558 \\
\precsim 274 / q 284 \\
15.6 \pm 1.4\end{array}$ & $\begin{array}{l}\text { PA (PE in school, how } \\
\text { often students exercised } \\
\text { with increased heart } \\
\text { rate more than 30min } \\
\text { in a week) }\end{array}$ & 5 & $13 \%$ \\
\hline $\begin{array}{l}\text { Florentino et al., } \\
2002 \\
\text { (Philippines) }^{31}\end{array}$ & $\begin{array}{l}\mathrm{N}=1208 ; \\
\widehat{\jmath} / \text { + equal; } \\
\text { Range: } 8-10\end{array}$ & $\begin{array}{l}\text { PA pattern } \\
\text { questionnaire } \\
\text { (frequency of exercise } \\
\text { only; no duration } \\
\text { noted) }\end{array}$ & 3 & $\begin{array}{l}23 \% \text { (assumption } \\
\text { that they were } \\
\text { active for at least } \\
30 \mathrm{~min} \text { ) }\end{array}$ \\
\hline $\begin{array}{l}\text { Gonzalez-Suarez } \\
\text { \& Grimmer- } \\
\text { Somers, 2009 } \\
\text { (Philippines) }\end{array}$ & $\begin{array}{l}\mathrm{N}=380 \\
\widehat{\jmath} 167 / q 213 \\
11.5 \pm 0.5\end{array}$ & $\begin{array}{l}\text { Filipino-Modified PA } \\
\text { Questionnaire for older } \\
\text { children (Delivers } \\
\text { Activity Levels) }\end{array}$ & 1 & $\begin{array}{l}\sim 6 \% \text { ( } 7 \text { or more } \\
\text { times MVPA/wk, } \\
\text { no duration } \\
\text { delivered, } \\
\text { inclusion of school } \\
\text { time) }\end{array}$ \\
\hline $\begin{array}{l}\text { Kaluski et al., } \\
2008 \text { (Israel) }^{27}\end{array}$ & $\begin{array}{l}\mathrm{N}=6274 ; \\
\partial^{\top} 3457 / \phi 2817 \\
14.7 \pm 1.6\end{array}$ & $\begin{array}{l}\text { Questionnaire on type, } \\
\text { frequency and duration } \\
\text { of PA }\end{array}$ & 2 & $47 \%$ \\
\hline $\begin{array}{l}\text { Khoo \& Al- } \\
\text { Shamli, } 2010 \\
(\text { Oman })^{33}\end{array}$ & $\begin{array}{l}\mathrm{N}=330 ; q 330 \\
16.4 \pm 0.49\end{array}$ & $\begin{array}{l}\text { Survey assessment of } \\
\text { levels of PA in leisure } \\
\text { time (type, duration, } \\
\text { frequency) }\end{array}$ & 2 & $\begin{array}{l}33.3 \%(\text { PA } 3-4 \\
d / \text { wk for } 60-90 \\
\text { min) }\end{array}$ \\
\hline $\begin{array}{l}\text { Li et al. } 2007 \\
(\text { China })^{47}\end{array}$ & $\begin{array}{l}\mathrm{N}=1760 ; \\
\jmath^{\pi} 880 / 9880 ; \\
\text { Range: } 11-17\end{array}$ & $\begin{array}{l}\text { Adolescent PA Recall } \\
\text { Questionnaire (time } \\
\text { spent on PA in average } \\
\text { week) }\end{array}$ & 4 & $84 \%$ \\
\hline $\begin{array}{l}\text { Liou \& Chiang, } \\
2004 \text { (Taiwan) }^{48}\end{array}$ & $\begin{array}{l}\mathrm{N}=463 ; \\
\delta^{\top} 249 / \phi 214 ; \\
10.8 \pm 1.1\end{array}$ & $\begin{array}{l}3 \text { day-Physical Activity } \\
\text { Level questionnaire } \\
\text { (record one of nine PA } \\
\text { levels every } 15 \mathrm{~min} \text { ) }\end{array}$ & 2 & $80 \%(420 \mathrm{~min} / \mathrm{wk})$ \\
\hline
\end{tabular}




\begin{tabular}{|c|c|c|c|c|}
\hline $\begin{array}{l}\text { Mak \& Day, } \\
2010 \text { (Hong } \\
\text { Kong) }\end{array}$ & $\begin{array}{l}\mathrm{N}=5692 ; \\
\nwarrow 12613 / q 3079 \\
14.8 \pm 1.0\end{array}$ & $\begin{array}{l}\text { Health Related } \\
\text { Behavior General } \\
\text { Survey (self-report of } \\
\text { PA participation) }\end{array}$ & 5 & $\begin{array}{l}25 \% \text { (no duration } \\
\text { considered; only } \\
\text { extracurricular PA } \\
\text { considered) }\end{array}$ \\
\hline $\begin{array}{l}\text { Shi et al., } 2006 \\
\text { (China) }\end{array}$ & $\begin{array}{l}\mathrm{N}=824 ; \\
\hat{\jmath} 431 / 9393 ; \\
\text { Range: } 12-14\end{array}$ & $\begin{array}{l}\text { Self-administered } \\
\text { questionnaire on PA }\end{array}$ & 5 & $\begin{array}{l}45.2 \% \text { (vigorous } \\
\text { PA, but no } \\
\text { duration given) }\end{array}$ \\
\hline $\begin{array}{l}\text { Wang et al., } \\
2006 \\
\text { (Singapore) }^{28}\end{array}$ & $\begin{array}{l}\mathrm{N}=780 \\
\jmath^{\top} 285 / q 482 \\
12.2 \pm 0.47\end{array}$ & $\begin{array}{l}\text { Modified self- } \\
\text { administered PA- } \\
\text { checklist ( } 7 \text { day recall } \\
\text { of type, frequency, } \\
\text { duration of PA) }\end{array}$ & 2 & $\begin{array}{l}60.1 \% \\
(>300 \mathrm{~min} / \mathrm{wk})\end{array}$ \\
\hline $\begin{array}{l}\text { Wang et al., } \\
2011, \\
\text { (Singapore) }^{29}\end{array}$ & $\begin{array}{l}\mathrm{N}=847 \\
\jmath^{\Uparrow} 298 /+9515 ; \\
\text { Range: } 10-16\end{array}$ & $\begin{array}{l}\text { Modified self- } \\
\text { administered PA- } \\
\text { checklist ( } 7 \text { day recall } \\
\text { of type, frequency, } \\
\text { duration of PA) }\end{array}$ & 2 & $\begin{array}{l}60.2 \% \\
(>300 \mathrm{~min} / \mathrm{wk})\end{array}$ \\
\hline $\begin{array}{l}\text { Wang et al., } \\
2011 \\
\text { (Singapore) }^{30}\end{array}$ & $\begin{array}{l}\mathrm{N}=3333 ; \\
\jmath_{1} 1257 / \not 1963 ; \\
\text { Range: } 10-18\end{array}$ & $\begin{array}{l}\text { Modified self- } \\
\text { administered PA- } \\
\text { checklist (7day recall of } \\
\text { type, frequency, } \\
\text { duration of PA) }\end{array}$ & 2 & $\begin{array}{l}59.6 \% \\
(>300 \mathrm{~min} / \mathrm{wk})\end{array}$ \\
\hline
\end{tabular}

Note. *objective instrument used as well; MVPA=moderate to vigorous PA

In 11 studies the applied guidelines were met by less than $50 \%$ of the examined samples. The WHO guideline (2010), ${ }^{37}$ which requires the highest PA participation per week could be applied in one study. ${ }^{46}$ Here, only $6 \%$ met this guideline.

Table 3 shows the outcome of studies that measured the weekly or daily time children and adolescents spent on PA.

Table 3. Time Spent on PA

\begin{tabular}{|c|c|c|c|}
\hline Study (country) & $\begin{array}{l}\text { N; Sex; } \\
\text { Age } \\
\text { (years) } \\
\pm \text { SD } \\
\end{array}$ & Instrument & $\begin{array}{l}\text { Average time spent on } \\
\text { PA per week or day }\end{array}$ \\
\hline $\begin{array}{l}\text { Chan et al., } 2003 \\
\text { (Hong Kong) }^{50}\end{array}$ & $\begin{array}{l}\mathrm{N}=201 \\
\text { गे } 79 / \not 122 \\
13.8 \pm 1.1\end{array}$ & $\begin{array}{l}\text { Modifiable Activity } \\
\text { Questionnaire for } \\
\text { Adolescents (leisure } \\
\text { time PA-frequency, } \\
\text { duration for last } 12 \\
\text { months) }\end{array}$ & $\begin{array}{l}\text { Boys: } 9.1 \pm 6.6 \mathrm{~h} / \mathrm{wk} \\
\text { Girls: } 2.2 \pm 3.4 \mathrm{~h} / \mathrm{wk} \\
\text { (excluding school time) }\end{array}$ \\
\hline
\end{tabular}




\begin{tabular}{|c|c|c|c|}
\hline 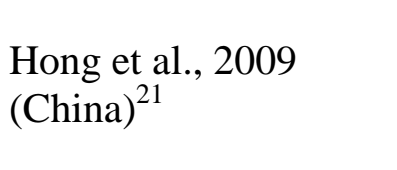 & $\begin{array}{l}\mathrm{N}=2444 \\
\widehat{\jmath} 1180 / \not 1264 \\
13.9 \pm 0.04\end{array}$ & $\begin{array}{l}\text { Questionnaire (time in } \\
\text { recreational PA, based } \\
\text { on IPAQ) }\end{array}$ & $9.8 \pm 7.8 \mathrm{~h} / \mathrm{wk}$ \\
\hline $\begin{array}{l}\text { Lachat et al., } 2008 \\
\text { (Vietnam)*32 }\end{array}$ & $\begin{array}{l}\mathrm{N}=227 \\
\partial / Q \text { no details; } \\
16\end{array}$ & $\begin{array}{l}\text { IPAQ and PAQA (PA } \\
\text { in normal week } \\
\text { recorded, many } \\
\text { activities included) }\end{array}$ & $\begin{array}{l}\text { IPAQ: } 45 \mathrm{~min} / \mathrm{d} \\
\text { PAQA: } 107 \mathrm{~min} / \mathrm{d} \\
\text { (MVPA considered } \\
\text { only) }\end{array}$ \\
\hline $\begin{array}{l}\text { Tudor-Locke et al., } \\
2003 \text { (Philippines) }{ }^{43}\end{array}$ & $\begin{array}{l}\mathrm{N}=1629 \\
\text { ग̄756/q873; } \\
15.6 \pm 0.5\end{array}$ & $\begin{array}{l}\text { PA (type, frequency, } \\
\text { duration during and } \\
\text { after school) }\end{array}$ & $\begin{array}{l}\text { Boys: } 6.8 \mathrm{~h} / \mathrm{wk} \\
\text { Girls: } 2.3 \mathrm{~h} / \mathrm{wk} \\
\text { (excluding commuting to } \\
\text { school) }\end{array}$ \\
\hline $\begin{array}{l}\text { Tudor-Locke et al., } \\
2003 \text { (China) }\end{array}$ & $\begin{array}{l}\mathrm{N}=2675 \\
\hat{\jmath} 1423 / \text {; } 1252 \\
11.5 \pm 3.3\end{array}$ & $\begin{array}{l}\text { PA survey on type, } \\
\text { frequency and duration } \\
\text { of PA (parents assisted } \\
\text { response by under } 10 \\
\text { yrs olds) }\end{array}$ & $\begin{array}{l}\text { Boys: } 45 \mathrm{~min} / \mathrm{d} \\
\text { Girls: } 38 \mathrm{~min} / \mathrm{d} \\
\text { (MVPA during \& } \\
\text { before/after school; } \\
\text { excluding commuting to } \\
\text { school) }\end{array}$ \\
\hline $\begin{array}{l}\text { Wickramasinghe et } \\
\text { al., } 2005 \text { (Sri Lanka) }\end{array}$ & $\begin{array}{l}\mathrm{N}=1102 ; \\
\precsim 485 / q 617 \\
\text { Range: } 8-12\end{array}$ & $\begin{array}{l}\text { Questionnaire } \\
\text { regarding PA behavior } \\
\text { (filled out by } \\
\text { guardians/parents) }\end{array}$ & $\begin{array}{l}\text { No PA: } 15.2 \% \\
<1 \mathrm{~h}: 22.1 \% \\
1-2 \text { h: } 51.1 \% \\
3-4 \text { h: } 8.3 \% \\
>5 \text { h: } 2.6 \% \text { (weekdays) }\end{array}$ \\
\hline $\begin{array}{l}\text { Zalilah et al., } 2006 \\
\text { (Malaysia) }^{11}\end{array}$ & $\begin{array}{l}\mathrm{N}=618 \\
\nwarrow 301 / q 317 \\
13.1 \pm 0.8\end{array}$ & $\begin{array}{l}\text { PA 3-day activity } \\
\text { record }\end{array}$ & $\begin{array}{l}2.8 \mathrm{~min} / \mathrm{d} \text { MVPA } \\
\text { (including housework } \\
\text { and other movements) }\end{array}$ \\
\hline
\end{tabular}

Note. *objective instrument used as well; MVPA=moderate to vigorous PA

Three studies reported data concerning weekly time spent with PA. ${ }^{21,43,50}$ School-time PA was excluded in two papers, ${ }^{21,50}$ whereas the study by Tudor-Locke et al. ${ }^{43}$ from the Philippines excluded only school related commuting activities. The data showed that younger adolescents spent approximately 2.5 hours more on PA than their older counterparts. Two of these studies conducted also separate analysis for male and female children or adolescents and showed that girls were consistently less active than boys. ${ }^{45,50}$ 
Daily PA time was measured in four studies, ${ }^{11,12,32,51}$ whereas the articles from Malaysia $^{11}$, China ${ }^{12}$ and Vietnam ${ }^{32}$ recorded MVPA. The time spent on PA varied greatly between these three countries, with $2.8 \mathrm{~min} / \mathrm{d}$ in Malaysia and $107 \mathrm{~min} / \mathrm{d}$ measured with the PAQA in Vietnam. Only one study displayed separate data for boys and girls, indicating that females are less active. ${ }^{12}$

Four studies used different subjective approaches. Mahfouz et al. ${ }^{13}$ found that approximately $31 \%$ of Saudi Arabian school-age (17.2 \pm 1.1 years) adolescents $(\mathrm{N}=1,869$; 1249 males and 620 females) did not engage in at least 30 minutes of PA in the past seven days. In Taiwan, Huang and Malina ${ }^{52}$ revealed that $32 \%$ of girls and 55\% of boys, aged $12-14$ years ( $\mathrm{N}=282 ; 138$ boys and 144 girls), were not moderately or vigorously active, based on the results of the 3-day Activity Recall Questionnaire. Henry et al. ${ }^{34}$ used the same questionnaire in the United Arab Emirates study with a female sample (11-16 years, N=58) and quantified the responses to a specific PA level of 1.26, representing an activity level lower than in other countries. ${ }^{53,54}$ In Hong Kong, Yu et al., ${ }^{55}$ conducted a study using the PAQ-C to examine the average level of habitual moderate to vigorous PA in schoolchildren $(10.36 \pm 1.71$ years). Activity levels were classified from 1 (lowest activity) to 5 (highest activity) with responses to eight PA behaviour questions averaged to provide a final score. The sample ( $\mathrm{N}=333 ; 189$ boys and 144 girls) measured an average level of 2.42, interpreted as medium activity.

\section{Objective measures of $P A$}

Eight studies used objective measures to obtain PA data of school-age children and adolescents with six studies using an accelerometer. ${ }^{10,26,32,35,43,56} \mathrm{Chia}^{42}$ utilized heart-rate measurements and a survey, whereas Corder et al. ${ }^{26}$ used the DLW method, accelerometry, and a questionnaire. See Table 4 for the results of objective measures. 
Table 4. PA Prevalence Measured with Objective Instruments

\begin{tabular}{|c|c|c|c|}
\hline Study (country) & $\begin{array}{l}\mathrm{N} ; \mathrm{Sex} ; \text { Age } \\
\text { (years) } \pm \mathrm{SD}\end{array}$ & Device & Outcome \\
\hline $\begin{array}{l}\text { Chia, } 2008 \\
\text { (Singapore) }^{*^{42}}\end{array}$ & $\begin{array}{l}\mathrm{N}=280 \\
\circlearrowright 140 / \not 140 ; \\
\text { Gp 1: } 10.6 \pm 0.3 \\
\text { Gp 2: } 14.8 \pm 0.6\end{array}$ & $\begin{array}{l}\text { Polar Vantage NV } \\
(10 \text { hours per day } \\
\text { on } 2 \text { weekdays } \\
\text { and } 1 \text { weekend } \\
\text { day) }\end{array}$ & $\begin{array}{l}\text { Inactive }(\mathrm{HR}<120 \mathrm{bpm}): 90 \% \\
\text { Light active (HR:120-139 bpm): } \\
7 \% \\
\text { Moderate active (HR: } 140-159 \\
\text { bpm): } 1.5 \% \\
\text { Vigorous active (HR>160 bpm): } \\
1.5 \%\end{array}$ \\
\hline $\begin{array}{l}\text { Corder et al., } \\
2010 \text { (India)*26 }\end{array}$ & $\begin{array}{l}\mathrm{N}=30 \\
\widehat{\lambda} 15 / q 15 \\
15.8 \pm 0.6\end{array}$ & $\begin{array}{l}\text { DLW (urine } \\
\text { samples on } 7 \\
\text { consecutive days) }\end{array}$ & $\begin{array}{l}\text { PA energy expenditure: } \varnothing 53.0 \\
\mathrm{~kJ} / \mathrm{kg}_{\mathrm{FFM}} / \mathrm{d} \text { (daily average of } 7 \text { days) }\end{array}$ \\
\hline $\begin{array}{l}\text { Corder et al., } \\
2010 \text { (India)*26 }\end{array}$ & $\begin{array}{l}\mathrm{N}=30 \\
\jmath^{\lambda} 15 / q 15 \\
15.8 \pm 0.6\end{array}$ & $\begin{array}{l}\text { Accelerometer }[7 \\
\text { days, obtain } \\
\text { average } \\
\text { movement in } \\
\text { counts per minute } \\
(\text { cpm)] }\end{array}$ & $\begin{array}{l}\varnothing 337.7 \text { cpm }(\text { light active })^{\text {a }} \text {; } \\
\text { MVPA=37.5 min/d (average of } \\
\text { whole group); } \\
\text { More than } 60 \text { min MVPA per day: } \\
31 \% \text { in boys; } \\
\text { More than } 60 \text { min MVPA per day: } \\
0 \% \text { in girls }\end{array}$ \\
\hline $\begin{array}{l}\text { Lachat et al., } \\
2008 \\
(\text { Vietnam) }\end{array}$ & $\begin{array}{l}\mathrm{N}=227 ; \AA / Q \text { no } \\
\text { information; } \\
16\end{array}$ & $\begin{array}{l}\text { Accelerometer ( } 8 \\
\text { days, total counts } \\
\text { and time of } \\
\text { MVPA measured) }\end{array}$ & $\begin{array}{l}\varnothing \mathrm{MVPA}=28 \mathrm{~min} / \mathrm{day} ; \\
\text { MPA for at least } 30 \mathrm{~min} / \mathrm{day}=46 \% \text {; } \\
\text { MPA for at least } 60 \mathrm{~min} / \mathrm{day}=16 \% \text {; } \\
\varnothing 281,272 \mathrm{cpd}(\text { moderately active) }\end{array}$ \\
\hline $\begin{array}{l}\text { Nguyen et al., } \\
2010 \\
\text { (Vietnam) }^{10}\end{array}$ & $\begin{array}{l}\mathrm{N}=617 \\
\uparrow 333 / q 284 \\
13.9 \pm 0.7\end{array}$ & $\begin{array}{l}\text { Accelerometer }(7 \\
\text { days, average time } \\
\text { spent with MVPA } \\
\text { calculated) }\end{array}$ & $\varnothing \mathrm{MVPA}=60.3 \mathrm{~min} / \mathrm{day}$ \\
\hline $\begin{array}{l}\text { Rowlands et } \\
\text { al., } 2002 \text { (Hong } \\
\text { Kong) } 55\end{array}$ & $\begin{array}{l}\mathrm{N}=50 \\
\jmath^{\lambda} 24 / q_{2} 26 \\
9.1 \pm 0.9\end{array}$ & $\begin{array}{l}\text { Accelerometer [5 } \\
\text { weekdays and } 1 \\
\text { weekend day, } \\
\text { obtain average } \\
\text { movement in } \\
\text { counts per day } \\
(\text { cpd)] }\end{array}$ & $\begin{array}{l}\varnothing 299,407 \pm 147,075 \mathrm{cpd} \\
\text { (moderately active) (averaged over } \\
5 \text { weekdays) }\end{array}$ \\
\hline 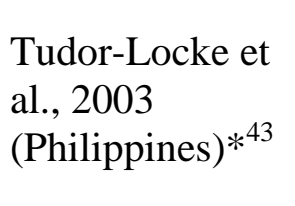 & $\begin{array}{l}\mathrm{N}=1629 \\
\nwarrow 756 / q 873 \\
15.6 \pm 0.6\end{array}$ & $\begin{array}{l}\text { Accelerometer (1 } \\
\text { day, energy } \\
\text { expenditure on PA } \\
\text { of } 1 \text { day recorded) }\end{array}$ & $\begin{array}{l}\mathrm{EE}_{\mathrm{PA}(24 \mathrm{~h})}=378 \pm 164.5 \mathrm{kcal} \text { (male) } \\
\mathrm{EE}_{\mathrm{PA}(24 \mathrm{~h})}=271 \pm 105.4 \mathrm{kcal} \text { (female) }\end{array}$ \\
\hline $\begin{array}{l}\text { Yamauchi et } \\
\text { al., } 2007 \\
\text { (South Korea, } \\
\text { China) })^{35}\end{array}$ & 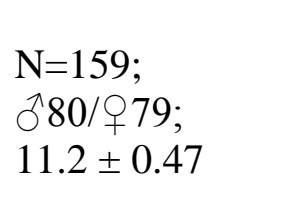 & $\begin{array}{l}\text { Accelerometer }(7 \\
\text { days, provide } \\
\text { TEE, STP and } \\
\text { PAL) }\end{array}$ & $\begin{array}{l}\text { STP }=10,946 \text { (male: } 11,388 \text {; female: } \\
\text { 10,504) (below cut offs) }{ }^{\mathrm{b}} \\
\text { TEE=1.54 kcal/d (male: } 1.5 ; \\
\text { female: } 1.57 \text { ) }\end{array}$ \\
\hline
\end{tabular}


Note. *subjective instrument used as well; ${ }^{a}=$ based on thresholds of Freedson et al. $(1998)^{57}$; ${ }^{\mathrm{b}=}$ cut offs (boys: 15000; girls: 12000) of Tudor-Locke et al. (2004) ${ }^{58}$; $\mathrm{EE}=$ Energy Expenditure; FFM=fat free mass; $\mathrm{MVPA}=$ moderate to vigorous PA; PAL=Physical Activity Level; STP=Daily step frequency; TEE=Total Energy Expenditure; $\varnothing=$ Arithmetic mean

PA, regardless of the measurement tool, is mostly low except in the study of Nguyen et al. ${ }^{10}$ where the sample shows an average participation of $60.3 \mathrm{~min} / \mathrm{d}$. In the Singaporean sample, $90 \%$ were inactive on weekdays according to the recorded heart rates. Indian schoolage youth are mostly light active. ${ }^{26}$ MVPA measured with an accelerometer in the Vietnam study by Lachat et al. ${ }^{32}$ is on average $28 \mathrm{~min} / \mathrm{d}$, indicating a high prevalence of physical inactivity according to the international guidelines. Boys and girls from China and South Korea showed daily step frequencies below the established cut offs. ${ }^{35}$

PA participation seems to decrease with age. Studies with older samples ${ }^{17,36}$ describe PA participation ranging from $28 \mathrm{~min} / \mathrm{d}$ to $37.5 \mathrm{~min} / \mathrm{d}$ compared to $60.3 \mathrm{~min} / \mathrm{d}$ in the younger sample studied by Nguyen et al. ${ }^{10}$ The three studies which displayed separate data for males and females illustrate lower PA participation for girls ${ }^{26,35,43}$ except for TEE in the research of Yamauchi et al. ${ }^{35}$

Two studies indicate the trend that higher levels of PA are associated with lower participation rates. ${ }^{32,42}$ Ninety percent of Singaporean youth is inactive, whereas $7 \%$ are light active and MVPA was observed in $3 \%$ (weekdays). ${ }^{42}$ Similarly, the research from Vietnam showed that $46 \%$ of the studied sample are at least $30 \mathrm{~min} / \mathrm{d}$ active compared to $16 \%$ who are active for about $60 \mathrm{~min} / \mathrm{d} .^{32}$

\section{Discussion}

Asia is the most populated continent in the world with a population of around 4 billion in 48 countries. Asia also shows rapid increases in obese and overweight populations and other health issues related to inactive lifestyles. Yet, only 14 countries (29\%) have published data concerning the PA prevalence of Asian school-age children and adolescents and the results of 
those studies are cause for concern. Although various measuring tools were used to collect PA data, nearly all data indicated that the majority of Asian children did not meet the guidelines for PA. Less than $50 \%$ of the samples reviewed met the minimum PA recommendations of 30 minutes, 3 days per week. Compared to other parts of the world, Asian school-age youth show low PA levels. Gorely et al. found that adolescents in the United Kingdom are more active with $63 \%$ of males participating in PA for at least 60 min per day. ${ }^{59}$ Higher participation rates are also found in Greek-Cypriot youth. More than 52\% of this population met the one hour per day PA guideline and can be considered as active. ${ }^{60}$ Similar rates in Asia can only be found in the studies of Wang et al. in Singapore, ${ }^{28-30}$ and Liou and Chiang ${ }^{48}$ in Taiwan. One major reason for the low activity levels in Asian school-age youth might be that most Asian countries are still developing and are hence more concerned about formal education programming that advances the country's reputation, enhances its economic status, and less concerned about the PA prevalence of its young people. ${ }^{61}$ School-age youth spent a considerable amount of time on school-based activities. ${ }^{29}$ Time that could be spent in PA, is usually taken up with homework or tuition requirements, which are considered more meaningful and more beneficial than PA.. ${ }^{56}$ Secondly, using PA as a health promotion method is much more recognized in westernized countries as seen in a longer history of various school based interventions concentrating on extracurricular PA. ${ }^{62}$ The WHO published a manuscript on PA interventions in school settings that indicates a minority of programs coming from non-developed countries especially in Asia. ${ }^{62}$ Thirdly, the wide range of activity rates in Asian adults could influence activity in youth. A recent study by Macniven et al. ${ }^{63}$ found that $7 \%$ to $93 \%$ of adults in Asia are sufficiently active. Even though different measurement methods were applied, low activity rates can be noted in many countries. A low PA level in adults could result in a limited role model behavior for younger people. Young people who do not perceive a certain amount of PA performed by their parents or other significant social units will be less likely to be active themselves. ${ }^{64,65}$ The resulting lack of PA 
is most likely to transfer into adulthood ${ }^{66}$ and can lead to the next generation ofless physically active adults who then effects PA attitudes of the upcoming generation of schoolage youth.

Another finding is that female youth in all the countries are less physically active than their male counterparts according to the data in the original research articles. This applies not only for Asia but seems to be a global phenomenon throughout all age groups as many international studies also came to the same conclusion. ${ }^{67,68}$

The review of articles revealed also that PA decreases with age. Younger youth have higher PA participation levels compared to the older ones. ${ }^{17,26}$ The trend of reduced PA with increasing age is also prevalent in other regions of the world, for example in Greece ${ }^{60}$ and the USA. $^{69}$ More formal responsibilities (e.g. in school) and a shift in individual preferences concerning leisure time activities causes the reduced participation in PA in adolescence on a global scale.

Even though every article in this review came to a conclusion about the PA participation of Asian school-age youth, it is not possible to make a generalization due to certain limitations. The use of 21 different measurement methods (18 subjective; 3 objective) applied in the original research papers is one of the major threats of this review. Subjective methods were the main data acquisition strategy as huge samples can be assessed in a time and cost effective way. However Armstrong et al. ${ }^{70}$ pointed out that children are oftentimes not able to recall activity periods and quantify them, as they are usually only sporadically active. Objective devices eliminate the weaknesses of the subjective methods, but are also costly, time consuming and sometimes inconvenient to conduct on the sample. These devices also do not provide any information about the type of activity. ${ }^{70}$ A comparison between the different objective and subjective data is difficult because every method uses a different approach. Prince et al. ${ }^{71}$ noted that studies which used both subjective and objective 
measurements showed a weaker correlation and influenced the findings, as shown in the studies included in this review.

Secondly, different studies used different definitions or dimensions of PA resulting in varying analysis procedures. Some studies only measured certain types of PA, whereas others were more comprehensive. Exclusion ${ }^{36,50}$ or inclusion of for example in-school-PA can cause immense differences in the final results leading to inaccurate conclusions.

Even though a careful literature review was undertaken, grey literature might be still unexplored. Despite the stated limitation this paper can serve as a baseline that provides a comprehensive picture about the PA prevalence of children and adolescents in Asia.

\section{Conclusion}

The data reviewed in this article support the contention that our target group generally has low levels of PA. But differences in age group, sex, ethnicity, study quality, and the use of 21 different instruments in the studies makes it almost impossible to provide an accurate picture or make comparisons. There is a crucial need for a standardized, reliable, and economically viable instrument that can accommodate differences in age, sex, and cultural subgroups in Asia to obtain unequivocal data that can inform any conclusion about PA prevalence of children and adolescents in Asia.

The authors recommend that education policy makers, administrators, and teachers use the information of low PA levels of school-age youth reported in this review to create activities designed to promote PA within the target age group. Such activities can be presented to not only educators but also parents through workshops, presentations, and handson events wherein participants are informed of the positive benefits of PA, shown how to implement changes, and encouraged to incorporate PA strategies into school and daily living activities.

Finally, it is important to note, that the type of activity applied in physical education classes or outside school settings is not as relevant as achieving the amount of activity that the 
established guidelines recommend, because culture, age, gender and other factors impact the acceptance or un-acceptance of the type of activities. 


\section{References}

1. World Health Organization. Nutrition in adolescence: issues and challenges for the health sector. Geneva: World Health Organization; 2005a.

2. Finkelstein EA, Khavjou OA, Thompson H, Trogdon JG, Pan L et al. Obesity and severe obesity forecasts through 2030. Am J Prev Med. (2012); 42(6): 563-570.

3. Prentice AM. The emerging epidemic of obesity in developing countries. Int $J$ Epidemiol. (2006); 35(1): 93-99.

4. Low S, Chin MC \& Deurenberg-Yap M. Review on epidemic of obesity. Ann Acad Med Singapore. (2009); 38(1): 57-65.

5. World Health Organization. Global health risks: mortality and burden of disease attributable to selected major risks. Geneva: World Health Organization; 2009.

6. Popkin BM \& Doak CM. The obesity epidemic is a worldwide phenomenon. Nutr Rev. (1998); 56(4): 106-114.

7. World Health Organization, Public Health Agency of Canada. Preventing chronic diseases: a vital investment. Geneva: World Health Organization; 2005b.

8. Wang Y \& Lobstein T. Worldwide trends in childhood overweight and obesity. Int $J$ Pediatr Obes. (2006); 1(1): 11-25.

9. Asia Pacific Cohort studies Collaboration. The burden of overweight and obesity in the Asia-Pacific region. Obes Rev. (2007); 8(3): 191-196.

10. Nguyen THHD, Tang HK, Kelly P, Hidde P \& Dibley M. Association between physical activity and metabolic syndrome: a cross sectional survey in adolescents in Ho Chi Minh City, Vietnam. BMC Public Health. (2010); 10(1): 141-149.

11. Zalilah MS, Khor GL, Mirnalini K, Norimah AK \&. Ang M. Dietary intake, physical activity and energy expenditure of Malaysian adolescents. Singapore Med J. (2006); 47(6): 491-498.

12. Tudor-Locke C \& Ainsworth BM. Physical activity and inactivity in Chinese schoolaged youth: the China Health and Nutrition Survey. Int J Obes. (2003); 27(9): 10931099.

13. Mahfouz AA, Shatoor AS, Khan MY, Dafalla AA, Mostafa OA \& Hassanein A. Nutrition, physical activity, and gender risks for adolescent obesity in Southwestern Saudi Arabia. Saudi J Gastroenterol. (2011); 17(5): 318-322.

14. Laitinen J, Power C \& Järvelin M. Family social class, maternal body mass index, childhood body mass index, and age at menarche as predictors of adult obesity. Am J Clin Nutr. (2001); 74(3): 287-294.

15. Harper MG. Childhood obesity: strategies for prevention. Fam Community Health. (2006); 29(4): 288-298.

16. Ogden LL, Yanowski SZ, Caroll MD \& Flegal M. The epidemiology of obesity. Gastroenterology. (2007); 132(6): 2087-2102.

17. Wabitsch M. Overweight and obesity in European children: definition and diagnostic procedures, risk factors and consequence for later health outcome. Eur J Pediatr. (2000); 159(13): 8-13.

18. Cruz ML \& Goran MI. The metabolic syndrome in children and adolescents. Curr Diab Rep. (2004); 4(1): 53-62.

19. Wang G \& Dietz WH. Economic burden of obesity in youth age 6 to 17: 1979-1999. Pedriatrics. (2002); 109(5): 81-91.

20. McGuigan MR, Tatasciore M, Newton R \& Pettigrew S. Eight weeks of resistance training can significantly alter body composition in children who are overweight or obese. J Strength Cond Res. (2009); 23(1): 80-85. 
21. Hong X, Li JQ, Xu E, Tse LA, Liang YQ, Wang ZY, et al. Physical activity inversely associated with the presence of depression among urban adolescents in regional China. BMC Public Health. (2009); 9(148): 1-9.

22. Janssen I \& LeBlanc AG. Systematic review of the health benefits of physical activity and fitness in school-aged children and youth. Int J Behav Nutr Phys Act. (2010); 7(1): 40.

23. Chen LJ, Haase AM \& Fox KR. Physical activity among adolescents in Taiwan. Asia Pac J Clin Nutr. (2007); 16(2): 354-361.

24. Sallis JS, Prochaska JJ \& Taylor WC. A review of correlates of physical activity of children and adolescents. Med Sci Sports Exerc. (2000); 32(5): 963-975.

25. Horst K, Paw MJCA, Twisk JWR \& Mechelen W. A brief review on correlates of physical activity and sedentariness in youth. Med Sci Sports Exerc. (2007); 39(8): 1241-1250.

26. Corder K, Brage S, Wright A, Ramachandran A, Snehalatha G, Yamuna A et al. Physcial activity energy expenditure of adolescents in India. Obesity. (2010); 18(11): 2212-2219.

27. Kaluski DN, Mezengia GD, Shimony T, Goldsmith R \& Berry EM. Prevalence and determinants of physical activity and lifestyle in relation to obesity among schoolchildren in Israel. Public Health Nutr. (2008); 12(6): 774-782.

28. Wang CKJ, Chia YHM Quek J \& Liu WC. Patterns of physical activity, sedentary behaviours and psychological determinants of physical activity among Singaporean school children. Int J Sport Exerc Psychol. (2006); 4(3): 227-249.

29. Wang CKJ, Biddle SJH, Liu WC \& Lim BSC. A latent profile analysis of sedentary and physical activity patterns. J Public Health. (2011); DOI: 10.1007/s10389-0110464-9.

30. Wang CKJ, Koh KT, Biddle SJH, Chia W \& Chye S. Physical activity patterns and psychological correlates of physical activity among Singaporean primary, secondary, and junior college students. ICHPER-SD J Res. (2011); 6(2): 3-9.

31. Florentino RF, Villavieja GM \& Lana RD. Dietary and physical activity pattern of 8to 10 year old urban schoolchildren in Manila, Philippines. Food Nutr Bull. (2002); 23(3): 267-273.

32. Lachat CK, Verstraeten R, Khanh LNB, Hagströmer M, Khan NC, Anh Van ND, et al. (2008). Validity of two physical activity questionnaires (IPAQ and PAQA) for Vietnamese adolescents in rural and urban areas. Int J Behav Nutr Phys Act. (2008); 5 (1): 37-44.

33. Khoo S \& Al-Shamli AK. Leisure-time physical activity and physical fitness of male adolescents in Oman. Asia Pac $J$ Public Health. (2010); DOI: 10.1177/1010539510366178.

34. Henry CJK, Lightowler HJ \& Al-Hourani HM. Physical activity and levels of inactivity in adolescent females ages 11-16 years in the United Arab Emirates. Am J Hum Biol. (2004); 16(3): 346-353.

35. Yamauchi T, Kim SN, Lu Z, Ichinaru N, Maekuwa R, Natsuhara K, et al. Age and gender differences in the physical activity pattern of urban schoolchildren in Korea and China. J Physiol Anthropol. (2007); 26(2): 101-107.

36. Mak KK \& Day JR. Secular trends of sports participation, sedentary activity and physical self-perception in Hong Kong adolescents, 1995-2000. Acta Paediatr. (2010); 99(11): 1731-1734.

37. World Health Organization. Global recommendations on physical activity for health. Geneva: World Health Organization; 2010. 
38. U.S Department of Health and Human Service and U.S Department of Agriculture. Dietary guidelines for Americans, 2005. $6^{\text {th }}$ Ed, Washington, DC.: U.S. Government Printing Office; 2005.

39. U.S. Department of Health and Human Service. Healthy people 2010. Physical activity and fitness. Washington, D.C.: U.S. Government Printing Office; 2000.

40. Strong WB, Malina RM, Blimkie CJR, Daniels SR, Dishman RK, Gutin SR et al. Evidence Based Physical Activity For School-Age Youth. J Pediar. (2005); 146(6): $732-737$.

41. Kowalski K, Crocker PRE \& Donen RE. The physical activity questionnaire for older children $(P A Q-C)$ and adolescents (PAQ-A) manual. College of Kinesiology, University of Saskatchewan Canada; 2004.

42. Chia M. Physical inactivity among children and adolescents in Singapore: a paradoxial issue. Acta Kinesiol. (2008); 2(2): 7-15.

43. Tudor-Locke C, Ainsworth BE, Adair LS, Du S \& Popkin BM. Physical activity in Filipino youth: The Cebu Longitudinal Health and Nutrition Survey. Int J Obes. (2003); 27(2): 181-190.

44. Cho MH. The strength of motivation and physical activity level during leisure time among youth in South Korea. Youth \& Society. (2004); 35(4): 480-494.

45. Chou YC \& Pei JS. Risk factors of adolescent obesity in Taiwan and its association with physical activity, blood pressure and waist circumference. Asian J Sports Med. (2010); 1(4): 214-222.

46. Gonzalez-Suarez CB \& Grimmer-Somers K. Physical activity pattern of prepubescent Filipino school children during school days. J Sch Health. (2009); 79 (7): 304-311.

47. Li M, Dibley MJ, Sibbritt DW, Zhou X \& Yan H. Physical activity and sedentary behaviour in adolescents in Xi'an City, China. J Adolesc Health. (2007); 44(1): 99101.

48. Liou YM \& Chiang LC. Levels of physical activity among school-age children in Taiwan: a comparison with international recommendations. J Nurs Res. (2004); 12(4): 307-316.

49. Shi Z, Lien N, Kumar BN \& Holmboe-Ottesen G. Physical activity and associated socio-demographic factors among school adolescents in Jiangsu Province, China. Prev Med. (2006); 43(3): 218-221.

50. Chan EW, Au EYM, Chan BHT, Kwan MKM, Yiu PYP \& Yeung EW. Relations among physical activity, physical fitness, and self-perceived fitness in Hong Kong adolescents. Percept Mot Skills. (2005); 96: 787-797.

51. Wickramasinghe VP, Lamabadusuriya SP, Atapattu N, Sathyadas G, Kuruparananthan S \& Karunarathne P. Dietary and physical activity pattern of school children in an urban area of Sri Lanka. Sri Lanka Journal of Child Health. (2005); 34(2): 44-49.

52. Huang YC \& Malina RM. Physical activity and health related physical fitness in Taiwanese adolescents. J Physiol Anthopol Appl Human Sci. (2002); 21(1): 11-19.

53. Ekelund U, Aman J, Yngve A, Renman C, Westerterp K \& Sjostrom M. Physical activity but not energy expenditure is reduced in obese adolescents: a case-control study. Am J Clin Nutr. (2002); 76(5): 935-941.

54. Huang YC \& Malina RM. Physical activity and correlates of estimated energy expenditure in Taiwanese adolescents 12-14 years of age. Am J Hum Biol. (1996); 8(2): 225-236.

55. Yu CCW, Chan S, Cheng F, Sung RYT \& Hau KT. Are Physical activity and academic performance compatible? Academic achievement conduct, physical activity and self esteem of Hong Kong Chinese primary school children. Educ Stud. (2006); 32(4): 331-341. 
56. Rowlands AV, Eston RG, Louie L, Ingledew DK, Tong KK \& Fu FH. Physical activity levels of Hong Kong Chinese children: relationship with body fat. Pediatr Exerc Sci. (2002); 14(3): 286-296.

57. Freedson PS, Melanson E \& Sirard J. Calibration of the computer science and applications, inc. accelerometer. Med Sci Sports Exerc. (1998); 30(5), 777-781.

58. Tudor-Locke C, Pangrazi RP, Corbin CB, Rutherford WJ, Vincent SD, Raustorp A, et al. BMI-referenced standards for recommended pedometer-determined steps/day in children. Prev Med. (2004); 38(6): 857-864.

59. Gorely T, Marshall SJ, Biddle SJH \& Cameron N. The prevalence of leisure time sedentary behaviour and physical activity in adolescent girls: an ecological momentary assessment approach. Int J Pediatr Obes. (2007); 4(4), 289-298.

60. Loucaides CA, Jago R \& Theophanous, M. Physical activity and sedentary behaviours in Greek-Cypriot children and adolescents: a cross-sectional study. Int J Behav Nutr Phys Act. (2011); 8(1), 90-101.

61. Booth A. Education and economic development in Asia: myths and realities. ASEAN Econ Bull. (1999); 16(3), 290-306.

62. World Health Organization. Interventions on diet and physical activity: what works: summary report. Geneva: World Health Organization; 2009.

63. Macniven R, Bauman A \& Abouzeid M. A review of population-based prevention studies of physical activity in adults in the Asia-Pacific region. BMC Public Health. (2012); 12(1), 41-51.

64. Sallis JF, Prochaska JJ \& Taylor WC. A review of physical activity of children and adolescents. Med Sci Sports Exerc. (2000); 32(5), 963-975.

65. Biddle SJH, Atkin AJ, Cavill N \& Foster C. Correlates of physical activity in youth: systematic review of quantitative systematic reviews. Int J Sports Exerc Psy. (2011); 4(1), 25-49.

66. Telama R, Yang X, Viikari J, Välimäki I, Wanne O et al. Physical activity from childhood to adulthood: a 21-year tracking study. Am J Prev Med. (2005); 28(3), 267273.

67. Eisenmann JC, Katzmarzyk PT \& Tremblay MS. Leisure-time physical activity Levels among Canadian adolescents, 1981-1998. J Phys Act Health. (2004); 1; 154-162.

68. Gordon-Larson P, Nelson MC \& Popkin BM. Longitudinal physical activity and sedentary behaviour trends: adolescents to adulthood. Am J Prev Med. (2004); 27(4), 277-283.

69. Nelson MC, Neumark-Stzainer D, Hannan PJ, Sirard JR \& Story M. Longitudinal and secular trends in physical activity and sedentary behaviour during adolescence. Pediatrics. (2006); 118(6), 1627-1632.

70. Armstrong N \& Welsman JR. The physical activity patterns of european youth with reference to methods of assessment. Sports Med. (2006); 36(12), 1067-1086.

71. Prince SA, Adamo KB, Hamel M, Hardt J, Connor-Gorber S \& Tremblay M. A comparison of direct versus self-report measures for assessing physical activity in adults: a systematic review. Int J Behav Nutr Phys Act. (2008); 5(1), 56-79. 